\title{
Editorial
}

\section{Early or Late Parenteral Nutrition in Critically Ill Children: Practical Implications of the PEPaNIC Trial}

\author{
Olivier Goulet ${ }^{a}$ Frank Jochum ${ }^{b}$ Berthold Koletzko ${ }^{c}$

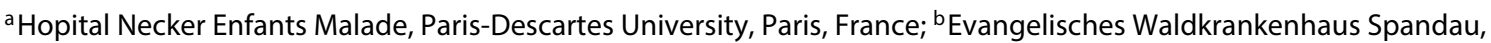 \\ Berlin, and 'Ludwig-Maximilians-Universität Munich, Dr. von Hauner Children's Hospital, Munich, Germany
}

Observational data from patients treated in pediatric intensive care units (PICU) suggest that optimized nutritional care may improve clinical outcomes, particularly in patients with malnutrition or high nutritional risks [1-4]. Supplemental parenteral nutrition (sPN) is widely used in those patients where early enteral nutrition (EN) is not feasible or does not suffice to reach nutritional targets [5]. The benefit and safety of early sPN in critically ill adults and children has recently been questioned based on findings from studies in adults [6-9].

This important issue has been addressed by Fivez et al. [10] in an impressive, large randomized controlled multicenter trial. This PEPaNIC trial was conducted in 3 PICUs (Leuven, Belgium; Rotterdam, Netherlands; and Edmonton, Canada) and enrolled 1,440 pediatric surgical and medical ICU patients who were randomized to receive $\mathrm{SPN}$ in addition to EN, either as early PN (EarlyPN, $n=723$ ) or late PN (LatePN, $n=717$ ). EarlyPN was initiated within $24 \mathrm{~h}$ after ICU admission, supplementing EN aiming to meet macronutrient and caloric targets following each center's individual concepts. LatePN started on the morning of day 8 in patients receiving $<80 \%$ of caloric target from EN. Compared with the EarlyPN group, the LatePN group showed less new infections $(p<0.001)$, in particular, airway and bloodstream infections $(p=0.002)$, and a PICU stay shortened by a mean 2.7 days $(p=0.002)$. However, since the study was not blinded, it seems possible that the decision on ICU discharge (primary out-

\section{KARGER}

๑) 2017 S. Karger AG, Basel

E-Mail karger@karger.com

www.karger.com/anm come) may have been biased. A 3 days shorter duration of mechanical ventilation $(p=0.01)$ and a 4.1 day shorter hospital stay $(p=0.005)$ were also observed with EarlyPN. Mortality was similar in both groups, but hypoglycemia was more frequent with LatePN (9.1\%) vs. EarlyPN (4.8\%, $p=0.001)$. The authors conclude that withholding PN for 1 week is advantageous in critically ill children.

Based on the previous experience with the EPaNIC trial in adult ICU patients [6], we consider it likely that these findings will invigorate the controversy about potential harm of early sPN. However, several important points and questions related to the PEPaNIC trial arise, including the selection and heterogeneity of included patients and the choices taken regarding nutrition and glucose control management.

\section{Important Points and Questions}

\section{Heterogeneity of the Patient Population Included}

To achieve sufficient statistical power, 1,440 critically ill children had to be enrolled into the PEPaNIC trial. Recruiting such a large sample of PICU patients is a major achievement, even with the combined efforts of 3 large centers. To reach this large number of enrolled patients within a reasonable time, considerable heterogeneity of included patient had to be accepted (Table 1). Ages of included patients ranged from newborns to 17 years, al- 
Table 1. Important points arising from the PEPaNIC trial

In a very large, unblinded randomized multicenter trial in critically ill children, withholding sPN for 1 week was associated with improved clinical outcomes, including reduced incidence of new infections and duration of ICU dependency, compared to starting sPN on the first day of ICU stay

There was considerable heterogeneity of the patient population with regard to age and diagnoses, which may have influenced the results obtained and limited transferability to clinical practice

A large number of patients included in the trial appear to have received sPN without a strict indication based on undernutrition or not reaching adequate intake with EN, and hence overuse of PN may have contributed to untoward effects

Nutrition and glucose management protocols were not standardized across centers, which may have influenced outcomes

It is likely that caloric overfeeding occurred in the early PN group which may have contributed to the higher incidence of adverse effects

though preterm neonates were excluded. Given that critically ill infants, children, and adolescents present with widely variable metabolic conditions and nutritional requirements, there are large differences in practices of applying PN $[11,12]$. A post hoc subgroup analysis of the 209 term infants aged less than 4 weeks of age showed similar or greater benefits for the primary outcomes, but no further subgroup analysis was reported for the age groups between 4 months and 17 years, which include the majority of enrolled patients.

The majority of included patients underwent elective surgery, predominantly cardiac surgery $(\sim 38 \%)$. Children undergoing emergency surgery, for example, traumatic brain injury, were also enrolled along with medical and cancer patients. Due to this further heterogeneity of the patient population, some children in the acute phase after traumatic injury or critical illness and emergency patients would have had a much more pronounced catabolic state than others such as elective surgery patients, resulting in very different caloric and protein requirements [13]. The results of the multivariable logistic regression analysis/ Cox proportional hazard analysis presented in the online supplementary material published by Fivez et al. [10] do not show any statistically significant interaction between treatment assignment and age/diagnostic group on the primary outcomes. This is rather surprising and challenges current clinical knowledge. Further post hoc analyses of the main study outcomes by age and diagnostic groups would seem desirable to assess the potential risk of bias more accurately. In addition, the effects of underlying diseases and their severity and of concomitant treatments on outcomes, and their possible interaction with sPN would also be of interest.

Early or Late PN in Critically Ill Children: Practical Implications of the PEPaNIC Trial

\section{Potential Center Effects}

Local protocols for initiation of PN in the EarlyPN group as well as the macronutrient and caloric targets to be achieved differed considerably between the 3 centers. Apparently, this allowed for conducting the study without the need for major changes in local routines. As a consequence, there was considerable variability in nutritional care with regard to targeted energy and macronutrient intakes, start of amino acid and lipid supply, and choice of parenteral lipid emulsions. Moreover, the 3 centers used different approaches to determine energy requirements. Energy expenditure was measured in some but not in all children treated in Edmonton. Only the center in Rotterdam appears to have adhered to the ESPGHAN/ESPEN PN guidelines [11] for setting parenteral macronutrient and caloric intake targets. There was also considerable variability with regard to blood glucose management across participating centers. A rather tight protocol for glycemic control was applied in Leuven, whereas Rotterdam and particularly Edmonton were more liberal in that aspect. It remains unclear whether children receiving the different nutritional strategies were equally distributed among EarlyPN and LatePN groups. Thus, it would appear highly relevant to assess centerdependent influences on the reported outcomes in detail.

\section{Indication for Starting sPN in the Study Patients}

The risk of malnutrition was estimated using the "Screening Tool for Risk on Nutritional Status and Growth" (STRONGkids) score. Children with a score $\geq 2$ were eligible for study participation. STRONGkids is a nutritional screening tool for hospitalized children based on a questionnaire that does not include any anthropo- 
metric measures [14-17]. The method has not been validated for critically ill children. The aim of the screening tool is to identify children with an increased for current or future malnutrition, and to select them for further diagnostic evaluation, but there is no reason to conclude that such a screening tool score would provide a valid basis for the decision to initiate $\mathrm{sPN}$.

Unfortunately, changes in markers of nutritional status were not considered as end points of the PEPaNIC trial. The reported median body mass index (BMI) in both groups of $15 \mathrm{~kg} / \mathrm{m}^{2}$ is similar to the median BMI of healthy children at the average age of 1.4-1.5 years. Thus, it appears that most children enrolled into the study were not underweight. In clinical practice, the indication for sPN is not only based on BMI but also on the difference between nutrient needs and achieved intakes, taking into account the markers of the patient's metabolic condition such as body weight changes, blood glucose, triglyceride levels, and others. If part of the patients included in the PEPaNIC trial would not have required $\mathrm{sPN}$, this could certainly have altered the evaluation of benefit vs. risk.

In the EarlyPN group, sPN was started on the first day in all patients, regardless of indication, age, or tolerance for EN, which contrasts with current concepts of clinical care. Usually, EN is established as a first line of nutritional therapy whenever possible, and sPN is not initiated until 2-3 days later in case it turns out that adequate amount of energy and protein cannot be provided via the enteral route. The duration of PICU treatment was 4 days or less in approximately $50 \%$ of patients included in the PEPaNIC trial. Thus, in view of the short duration of critical illness and PIU stay, and the lack of a clear indication for PN, many of the included patients may not even have required sPN. The use of $\mathrm{PN}$ without a clear need may be detrimental and might possibly explain the worse outcome in the EarlyPN group. One also needs to consider that approximately $75 \%$ of patients assigned to the LatePN group had already been discharged from the PICU by day 7 and thus never received any PN.

These aspects question the transferability of the results reported by the PEPaNIC trial for standard clinical practice following current treatment standards $[11,12,18]$.

\section{Supply Relative to Needs}

Energy intakes in the patients enrolled into the PEPaNIC trial were based primarily on estimates and equations. Only in a subset of patients in the Edmonton center, energy intakes were based on the results of indirect calorimetry. Approximately $45 \%$ of patients were infants under the age of 1 year whose energy and nutrient requirements differ markedly from those in older children $[11,19]$. Yet, only in the Rotterdam center, parenteral energy and nutrient provision adhered to current guidelines [11]. Thus, it appears that some patients may have been under- or overfed with $\mathrm{sPN}$, which may have contributed to the poor outcome in the EarlyPN group. It would appear desirable to explore metabolic profiles and laboratory data, reflecting adequate glucose, amino acid, and lipid metabolism, to clarify this question.

\section{Are Adverse Effects Due to Early PN or Due to a Supply Exceeding Needs}

Patients were randomized to 2 predefined nutrition strategies, irrespective of individual conditions and needs. In contrast to current treatment standards $[11,12,18]$, EN was intentionally limited without attempting to advance intakes over time. PN was provided to an inhomogeneous group of patients, apparently with and without the need for PN. The question arises whether trying to reach an arbitrary nutritional target not related to individual needs could have led to a high rate of overfeeding, which may have contributed to the reported adverse outcomes. Moreover, data provided in the online supplementary material show rather low protein intakes while energy intakes were high, leading to a disturbingly low protein/energy ratio. An insufficient protein intake in combination with an inadequately low nitrogen/energy ratio may induce untoward effects.

\section{Possible Causes and Mechanisms for the Increased Rate of Infections}

$\mathrm{PN}$ may increase the risk of infection, especially when the daily care of the CVC is suboptimal as may occur in the reality of PICU settings with high staff workloads. Surprisingly, $37.8-39.7 \%$ of the PEPaNIC trial patients had infections already at admission. Unfortunately, detailed information on the types and duration of infections and on antibiotic resistance was not reported. The high rate of pre-existing infections may have made it more difficult to distinguish newly occurring infections. The underlying mechanisms for the reported increase in infections, in particular, respiratory or urinary tract infections, would be of interest to underpin a causal relationship to EarlyPN. It is noteworthy that bilirubin and $\mathrm{C}$-reactive protein plasma levels were significantly higher in the late PN group. Since other inflammatory and immunological parameters are not reported, the underlying causes remain unclear and deserve further evaluation. 


\section{Conclusions}

Defining and achieving optimal nutritional care of critically ill infants, children, and adolescents is a challenging goal. These patient groups tend to be very heterogeneous not only regarding age, but also regarding their pathology, nutritional and metabolic status, and nutritional needs. The PEPaNIC trial demonstrates that large, multicenter randomized trials on important questions on nutritional care can be realized which is very encouraging. However, the results achieved must be interpreted with caution, since a number of questions arise. The indiscriminate use of early $\mathrm{sPN}$ in addition to $\mathrm{EN}$ in patients who may not require $\mathrm{PN}$ may have adverse effects. Further subgroup analyses in relation to the main outcome parameters are warranted to enable a better understanding of susceptible subgroups, potential underlying mechanisms, and further the interpretation of the study results. Even though such a post hoc analysis would be associated with an increased risk of bias, it would be valuable considering the heterogeneity of the patients, and it may provide the basis for further trials addressing more specific questions. The findings of the PEPaNIC trial in children and of the EPaNIC trial in adults have not been replicated in trials with patients randomized based on their confirmed need of sPN after carefully evaluating the possibility of achieving nutritional targets with EN. Thus, it appears premature to extrapolate results to all critically ill patients and to generally withhold PN. Clinicians should be encouraged to initiate EN at a safe and efficient level and to subsequently advance EN as much as feasible. The conditions warranting the initiation of sPN in order to achieve an additional benefit deserve further careful exploration. In such future studies, the patients' individual metabolic situation should be considered in order to avoid overnutrition or insufficient nutrient supply.

\section{Acknowledgments}

The constructive help of Dr. K.S. Kuhn in drafting and revising the language of this manuscript is gratefully acknowledged.

\section{Disclosure Statement}

O.G., the Evangelisches Waldkrankenhaus Spandau and its employee F.J. and the Ludwig-Maximilians-Universität München and its employee B.K. have received support for scientific and educational activities from companies that market nutritional products.

\section{Funding Sources}

The work of B.K. is financially supported in part by the Commission of the European Communities, programmes Early Nutrition (FP7-289346), DYNAHEALTH (No. 633595), and the European Research Council Advanced Grant META-GROWTH (ERC-2012-AdG - No. 322605), and the German Ministry of Education and Research, Berlin (No. 01 GI 0825).

\section{References}

1 Mehta NM: Approach to enteral feeding in the PICU. Nutr Clin Pract 2009;24:377-387.

2 Mehta NM, Compher C; A.S.P.E.N. Board of Directors: A.S.P.E.N. Clinical Guidelines: nutrition support of the critically ill child. JPEN J Parenter Enteral Nutr 2009;33:260-276.

3 Mehta NM, Duggan CP: Nutritional deficiencies during critical illness. Pediatr Clin North Am 2009;56:1143-1160.

4 Mehta NM, Bechard LJ, Cahill N, Wang M, Day A, Duggan CP, et al: Nutritional practices and their relationship to clinical outcomes in critically ill children - an international multicenter cohort study*. Crit Care Med 2012;40:2204-2211.

5 Koletzko B, Bhatia J, Bhutta Z, Cooper P, Makrides M, Uauy R, et al: Pediatric Nutrition in Practice, 2nd, revised edition. Basel, Karger, 2015.

6 Casaer MP, Mesotten D, Hermans G, Wouters PJ, Schetz M, Meyfroidt G, et al: Ear-

Early or Late PN in Critically Ill Children: Practical Implications of the PEPaNIC Trial ly versus late parenteral nutrition in critically ill adults. N Engl J Med 2011;365:506517.

7 Heidegger CP, Berger MM, Graf S, Zingg W, Darmon P, Costanza MC, et al: Optimisation of energy provision with supplemental parenteral nutrition in critically ill patients: a randomised controlled clinical trial. Lancet 2013; 381:385-393.

8 Singer P, Anbar R, Cohen J, Shapiro H, Shalita-Chesner M, Lev S, et al: The tight calorie control study (TICACOS): a prospective, randomized, controlled pilot study of nutritional support in critically ill patients. Intensive Care Med 2011;37:601-609.

9 Doig GS, Simpson F, Sweetman EA, Finfer SR, Cooper DJ, Heighes PT, et al: Early parenteral nutrition in critically ill patients with short-term relative contraindications to early enteral nutrition: a randomized controlled trial. JAMA 2013;309:2130-2138.
10 Fivez T, Kerklaan D, Mesotten D, Verbruggen S, Wouters PJ, Vanhorebeek I, et al: Early versus late parenteral nutrition in critically ill children. N Engl J Med 2016;374:11111122.

11 Koletzko B, Goulet O, Hunt J, Krohn K, Shamir R: 1. Guidelines on Paediatric Parenteral Nutrition of the European Society of Paediatric Gastroenterology, Hepatology and Nutrition (ESPGHAN) and the European Society for Clinical Nutrition and Metabolism (ESPEN), supported by the European Society of Paediatric Research (ESPR). J Pediatr Gastroenterol Nutr 2005;41(suppl 2):S1-S87.

12 Koletzko B, Goulet O: Nutritional support in infants, children and adolescents; in Sobotka L (ed): Basics in Clinical Nutrition, ed 4. Prague, Gelén, 2011, pp 625-653.

13 Wischmeyer PE: The evolution of nutrition in critical care: how much, how soon? Crit Care 2013;17(suppl 1):S7. 
14 Hulst JM, Zwart H, Hop WC, Joosten KF: Dutch national survey to test the STRONGkids nutritional risk screening tool in hospitalized children. Clin Nutr 2010;29: 106-111.

15 Huysentruyt K, Alliet P, Muyshont L, Rossignol R, Devreker T, Bontems P, et al: The STRONG(kids) nutritional screening tool in hospitalized children: a validation study. Nutrition 2013;29:1356-1361.
16 Moeeni V, Walls T, Day AS: The STRONGkids nutritional risk screening tool can be used by paediatric nurses to identify hospitalised children at risk. Acta Paediatr 2014;103:e528-e531.

17 Spagnuolo MI, Liguoro I, Chiatto F, Mambretti D, Guarino A: Application of a score system to evaluate the risk of malnutrition in a multiple hospital setting. Ital J Pediatr 2013;39:81.

18 Agostoni C, Buonocore G, Carnielli VP, De Curtis M, Darmaun D, et al; ESPGHAN
Committee on Nutrition: Enteral nutrient supply for preterm infants: commentary from the European Society of Paediatric Gastroenterology, Hepatology and Nutrition Committee on Nutrition. J Pediatr Gastroenterol Nutr 2010;50:85-91.

19 Koletzko B: Nutritional needs of children and adolescents; in Sobotka L (ed): Basics in Clinical Nutrition, ed 4. Prague, Gelèn, 2011, pp 61-76. 